\title{
Role of cell based approaches in cancer immunotherapy
}

\begin{abstract}
Immunotherapies hold the potential for cancer treatment since their mode of action is distinct to chemo and radiation therapy and largely depends on harnessing body's own immune system. The major advantage associated with cancer immunotherapy is that cell responses are specific to tumor and with low or negligible toxicity. Preclinical and clinical studies have evidenced that modulation of immune system can subvert the immunosuppressive environment under progressive tumor conditions. The modulation can be brought into several ways including infusion of ex-vivo or in-vivo activated antigen presenting cells (dendritic cells), immune checkpoint antibodies, adoptive transfer of $\mathrm{T}$ cells, genetically modified $\mathrm{T}$ cells, cancer cell vaccines, stem cells, cytokines and others. In this review, we will keep the discussion focused to some of cell based approaches.
\end{abstract}

Keywords: immunotherapy, dendritic cells, adoptive $t$ cell therapy, mesenchymal stem cells, cancer
Volume 2 Issue 5 - 2017

\begin{abstract}
Anjum Mahmood,' Anjani Srivastava, ${ }^{2}$ Shivangi Srivastava, ${ }^{2}$ Hiteshree Pandya,' Neel Khokhani,' Divyang Patel,' Rangnath Mishra ${ }^{3}$ 'GIOSTAR Research Pvt Ltd, India

${ }^{2}$ Global Institute of Stem Cell Therapy and Research, USA

${ }^{3}$ Department of Medicine, National Jewish Health, USA
\end{abstract}

Correspondence: Rangnath Mishra, Department of Medicine, National Jewish Health, Denver, CO 80206, USA.

Email mishrar@njhealth.org

Received: February 17, 2017 | Published: May 05, 2017
Abbreviations: DCs, dendritic cells; MSCs, mesenchymal stem cells; MDSCs, myeloid derived suppressor cells; BBB, blood brain barrier; CAR, chimeric antigen receptor, IFN, interferon; TILs, tumor infiltrating lymphocytes; CSCs, cancer stem cells; PBMCs, peripheral blood mononuclear cells; IL, interleukin

\section{Introduction}

An integrated immune system prevents development and progression of neoplastic cells in a process termed as immune surveillance. T-cells play an important role in detecting and eliminating tumor cells. In turn, they are dependent on dendritic cells for tumor antigen presentation and activation signals to stimulate them. One of the most important reasons behind failure of cancer immunosurveillance is hampered T-cell activity due to lack of co-stimulatory activation signals by dendritic cells resulting into peripheral tolerance. Other factors driving tumor progression include immunosuppressive tumor micro-environment, infiltration of regulatory $\mathrm{T}$ cells, release of immunosuppressive cytokines like IL-10 and TGF- $\beta$, reduced expression of MHC molecules, myeloid derived suppressor cells (MDSCs) and heterogeneity of tumor sub-clones at the genetic level. Studies have shown that expansion of Treg cells is associated with poor prognosis and reduced survival. Similarly, abnormal accumulation of MDSCs is also correlated with tumor evasion mechanism. Though, chemotherapy is first line of treatment, the efficacy is restricted later due to development of drug resistance. The major reasons for resistance development includes drug-targeted gene amplification (e.g. BRAF gene) and substitution mutation in some cancer cells leading to the escape of drug cytotoxic effect. ${ }^{1}$ Further, non-specific cytotoxicity of chemo agents result into lymphodepliton. To address all these issues, new therapeutic interventions are required which alone or in combination alter the tumor microenvironment to enhance beneficial effects without causing toxicity. In this context, immunotherapy is expected to play significant role. Cancer immunotherapy can be defined as set of techniques aimed to eliminate malignant tumors through mechanisms involving immune system responses. The agents driving immune alteration are termed as immunomodulators. In this review, we will discuss briefly some of specific methods mediating immunomodulation including dendritic cell based approaches, adoptive $\mathrm{T}$ cells transfer and mesenchymal stem cells based targeted delivery of drugs.

\section{Dendritic cells}

The dendritic cells (DCs) based immunotherapeutic approach has emerged as one of alternative treatment options owing to its low toxicity in comparison to other standard methods. The clinical efficacy has been demonstrated with improvement in overall survival rate and low toxicity. The application of ex-vivo-generated DCs emerged in an effort to improve the therapeutic efficacy in cancer patients in whom the dysfunction of endogenous DCs is commonly observed. The DCs are generated using several approaches. The most common used method is ex-vivo differentiation of DCs from peripheral blood mononuclear cells (PBMCs) using interleukin-4 (IL-4) and granulocyte macrophage colony stimulating factor (GM-CSF). ${ }^{2}$ Other ways of generating ex-vivo DCs is to derive it from progenitor CD34+ cells or in-vivo stimulation using C-type lectin receptors (CLRs) present on DC surfaces. CLRs specific antibodies attached to tumor antigens are readily internalized by DCs and generate antigen specific antitumor immunity.,

In terms of clinical success, initially most of phase I/II studies demonstrated only safety and feasibility. The efficacy remained an issue in phase III due to inconsistent and inconclusive data. The first successful commercialized product was Sipuleucel-T for castration resistant prostate cancer. The vaccine was approved by FDA in $2010 .{ }^{5}$ The approval was based on phase III results of IMPACT (Immunotherapy Prostate Adenocarcinoma Treatment) trials. Later, several other studies demonstrated beneficial effect of DC immunotherapy in head and neck squaous cell carcinoma, ${ }^{6}$ uterus, ${ }^{7}$ prostate $^{8}$ and breast cancer and Her-2 positive ductal carcinoma insitu. ${ }^{9}$ However, for most cancers, preclinical success could not be translated up to phase III due inconstant data and less optimized process. 


\section{Adoptive t cell therapy}

Adoptive transfer of $\mathrm{T}$ cells is a potent treatment option for metastatic tumors. The $\mathrm{T}$ cell based interventions are specific, robust (undergoing upto 1000 fold clonal expansion) and retain memory. Further, $\mathrm{T}$ cells can infiltrate to the site of antigen and thus holds capacity to eradicate distant metastasis. In chimeric antigen receptor (CAR) approach $\mathrm{T}$ cells are engineered cells which provide specificity to the effector cells. Most of clinical investigations targeted B cell related malignancies using CD19 directed CART cells. These studies demonstrated response in many patients. ${ }^{10-12}$ Antigens like human epidermal growth factor receptor over-expressed in tumors like breast, ovarian, non small cell lung carcinoma (NSCLC), salivary gland, pancreatic and endometrial cancers are under investigation for CART cell development. ${ }^{13-17}$ Till now, most of the success of CART cells is limited around hematological malignancies, a huge scope is still available for exploring new antigens, directed to eliminate metastatic, resistant and non-hematological malignancies.

Tumor infiltrating lymphocytes (TILs) are found in the tumor region and are associated with anti-tumor activity. They are isolated from tumor, expanded under ex-vivo conditions, screened for antineoplastic activity and infused back into patients. Their presence in tumor is associated with better clinical outcome. These lymphocytes at tumor site recognize the antigens presented by MHCI and MHC II molecules on cell surfaces. TILs raised against melanoma recognize antigens especially MART-1, gp100 and tyrosinase. ${ }^{18-21}$ Another class of antigens termed as cancer/testis $(\mathrm{C} / \mathrm{T})$ antigens are also recognized by melanoma TILs. The class includes several antigens like MAGE, NYESO-1, RAGE, SAGE and SSX2.22,23 Rosenberg et al was pioneer in isolation and expansion of melanoma specific TILs developed for clinical purposes. ${ }^{24}$ Rosenberg et al.,${ }^{24}$ conducted three sequential clinical trials, in which 93 patients (metastatic melanoma) were treated with lympho depleting preparative regimen, autologous TILs and IL2. Objective response rates by RECIST criteria in the three trials were $49 \%, 52 \%$ and $72 \%$, respectively. Study showed that $22 \%$ of all patients achieved complete tumor regression and $19 \%$ of the patients were disease-free for more than three years. ${ }^{25}$ Till now, most of the clinical investigations focused on melanoma due to considerable success. However, non melanoma tumors demonstrated less feasibility due to lack of reproducibility of TIL generation from primary and metastatic tumors.

\section{Mesenchymal stem cells}

Mesenchymal stem cells (MSCs) are adult stem cells with unique characteristic ability of homing, facilitating their application in cancer immunotherapy. These adult stem cells are reported to migrate at site of inflammation, injury, infection and tumors where they immunomodulate the immediate micro-environment through secretion of soluble factors. ${ }^{26}$ The therapeutic value to MSCs is conferred by transportation of anti-tumor genes. MSCs act as delivery vehicle for many tumor inhibiting genes and factors to tumor site. ${ }^{27}$ They offer therapeutic advantage of ease of isolation, ex-vivo expansion, transduction and transplantation. The movement of MSCs to tumor site is driven by chemotactic factors, chemokines and chemo-attractants released by progressive tumors. ${ }^{28}$ MSCs hold another characteristic feature which makes them a favorable tool for carrying targeted anti-cancer gene i.e., they are immunoprivileged. The absence or low expression of MHC II, MHC I, CD80, CD40, and CD86 molecules on cell surface make them undetectable by host immune system. Further, immunoprivileged nature also confers possible use of allogenic MSCs. However, at same time, MSCs are immunosuppressive, which exerts significant effect on host disease. In case of graft versus host disease, transplant of MSCs offer a promising treatment, where disease can develop due to histo-compatibility mismatch. ${ }^{29}$ On other hand, application of MSCs can induce tumor progression due to immune inhibition.

Several genes demonstrating therapeutic efficacy in preclinical models have been tested for expression in MSCs as vehicle. The genes which have been engineered in MSCs to target tumor sites include IL-12, VEGFR-1, CX3CL1, HSV-Tk, TRAIL and IFN $\beta{ }^{30-33}$ Their expressions were related to localized and metastatic tumor inhibition and survival benefits in tumor models. HSV-Tk (herpes simplex virus thymidine kinase) is a pro drug converting enzyme which is delivered through MSCs along with systemic administration of ganciclovir. In this approach, which has been successfully tested in glioma and pancreatic cancer, MSCs carry the suicide enzymes to the tumor site thus avoiding systemic toxicity. ${ }^{34}$ In brain tumors like glioma where blood brain barrier (BBB) restrict passage of anti tumor therapy, MSCs based delivery of drugs can provide therapeutic solutions. ${ }^{35}$

\section{Future directions}

Recent advances in understanding the mechanism underlying tumor progression and role of immune system has laid the foundation of immunotherapy based interventions in clinical malignancies. By adopting unique immunotherapeutic approach specific to diseased condition and optimal conditions of delivery significant level of benefits can be expected. Further, exploration of new targeted strategies is also required to extend scope of application and avoid unwanted adverse events in patients. The targeting of other identified DC cell surface receptors like mannose receptor (MR), CIRE, DCSIGN, DCIR, LSECtin, L-SIGN, Langerin, Dectin, DNGR-1, MICL, MGL CLEC2, CLEC12B, LOX-1, BDCA-2, DEC205, scavenger receptor, DC-ASGPR, FIRE, DC-STAMP and Toll-like receptors (TLRs) will definitely open the new dimensions in in-vivo DC based approaches. ${ }^{5}$ Further, targeting of cancer stem cells (CSCs) via DCs will also improve specificity of anti-tumor activity. Similarly, role of MSC derived exosomes in delivery of therapeutic agents is also currently under investigation in several studies. Exosome-mediated delivery of tumor suppressor miRNAs and targeting of growthregulatory pathways, such as the Wnt and Hedgehog pathways, as well as angiogenic pathways, such as the VEGF and kinase pathways, could be novel strategies to monitor tumor growth. In light of current knowledge and advances in cancer immunotherapy we conclude that under optimal conditions, tangible benefits can be realized in cancer management.

\section{Acknowledgements}

None.

\section{Conflict of interest}

The author declares no conflict of interest.

\section{References}

1. Mahmood A, Rajasekar S, Bora C, et al. Synergistic Effect of dendritic cell vaccine with immune modulating chemo drugs. Journal of Academia and Industrial Research. 2015;3(12):590-597. 
2. O'Neill DW, Bhardwaj N. Differentiation of peripheral blood monocytes into dendritic cells. Curr Protoc Immunol. 2005; Chapter 22:Unit 22F, 4.

3. Banchereau J, Palucka AK, Dhodapkar M, et al. Immune and clinical responses in patients with metastatic melanoma to CD34+ progenitorderived dendritic cell vaccine. Cancer Res. 2001;61(17):6451-6458.

4. Turnis ME, Rooney CM. Enhancement of dendritic cells as vaccines for cancer. Immunotherapy. 2010;2(6):847-862.

5. Higano CS, Schellhammer PF, Small EJ, et al. Integrated data from 2 randomized, double-blind, placebo-controlled, phase 3 trials of active cellular immunotherapy with sipuleucel-T in advanced prostate cancer. Cancer. 2009;115(16):3670-3679.

6. Schuler PJ, Harasymczuk M, Visus C, et al. Phase I dendritic cell p53 peptide vaccine for head and neck cancer. Clin Cancer Res. 2014;20(9):2433-2444.

7. Coosemans A, Vanderstraeten A, Tuyaerts S, et al. Wilms' Tumor Gene 1 (WT1)-loaded dendritic cell imunotherapy in patients with uterine tumors:a phase I/II clinical trial. Anticancer Res. 2013;33(12):5495-5500.

8. Hildenbrand B, Sauer B, Kalis O, et al. Immunotherapy of patients with hormone-refractory prostate carcinoma pre-treated with interferongamma and vaccinated with autologous PSA-peptide loaded dendritic cells-a pilot study. Prostate. 2007;67(5):500-508.

9. Sharma A, Koldovsky U, Xu S, et al. HER-2 pulsed dendritic cell vaccine can eliminate HER-2 expression and impact ductal carcinoma in situ. Cancer. 2012;118(17):4354-4362.

10. Lee D, Kochenderfer J, Stetler-Stevenson M, et al. T cells expressing CD19 chimeric antigen receptors for acute lymphoblastic leukaemia in children and young adults: a phase 1 dose-escalation trial. Lancet. 2014;385(9967):517-528.

11. Gardner R, Jensen M. CD19CAR T cells: from humble beginnings to cancer immunotherapy's poster child. Cancer J. 2014;20:107-111.

12. Tasian SK, Gardner RA. CD19-redirected chimeric antigen receptormodified T cells: a promising immunotherapy for children and adults with B-cell acute lymphoblastic leukemia (ALL). Ther Adv Hematol. 2015;6(5):228-241.

13. Whilding LM, Maher J. ErbB-targeted CAR T-cell immunotherapy of cancer. Immunotherapy. 2015;7(3):229-241.

14. Scholl S, Beuzeboc P, Pouillart P. Targeting HER2 in other tumor types. Annals of Oncology. 2001;12(Suppl 1):S81-S87

15. Ahmed N, Brawley VS, Hegde M, et al. Human epidermal growth factor receptor 2 (HER2)-specific chimeric antigen receptor-modified T cells for the immunotherapy of HER2-positive sarcoma. J Clin Oncol. 2015;33(15):1688-1696.

16. Feng K, Guo Y, Dai H, et al. Chimeric antigen receptor-modified T cells for the immunotherapy of patients with EGFR-expressing advanced relapsed/refractory non-small cell lung cancer. Science China Life Sciences. 2016;59(5):468-479.

17. Almåsbak H, Aarvak T, Vemuri MC. CAR T cell therapy: A game changer in cancer treatment. Journal of Immunology Research. 2016;2016:10.

18. Bakker AB, Schreurs MW, de Boer AJ, et al. Melanocyte lineage-specific antigen gp100 is recognized by melanoma-derived tumor-infiltrating lymphocytes. J Exp Med. 1994;179(3):1005-1009.
19. Engelhard VH, Bullock TN, Colella TA, et al. Antigens derived from melanocyte differentiation proteins:self-tolerance, autoimmunity, and use for cancer immunotherapy. Immunol Rev. 2002;188:136-146.

20. Robbins PE, el-Gamil M, Kawakami Y, et al. Recognition of tyrosinase by tumor-infiltrating lymphocytes from a patient responding to immunotherapy. Cancer Res. 1994;54(12):3124-3126.

21. Romero P, Gervois N, Schneider J, et al. Cytolytic T lymphocyte recognition of the immunodominant HLA-A*0201-restricted Melan-A/ MART-1 antigenic peptide in melanoma. J Immunol. 1997;159(5):23662374.

22. Chen YT, Gure AO, Tsang S, et al. Identification of multiple cancer/testis antigens by allogeneic antibody screening of a melanoma cell line library. Proc Natl Acad Sci USA. 1998;95(12):6919-6923.

23. Scanlan MJ, Gure AO, Jungbluth AA, et al. Cancer/testis antigens: an expanding family of targets for cancer immunotherapy. Immunolog Rev. 2002; 188:22-32

24. Rosenberg SA, Packard BS, Aebersold PM, et al. Use of Tumor-Infiltrating Lymphocytes and Interleukin-2 in the Immunotherapy of Patients with Metastatic Melanoma. N Engl J Med. 1988;319(25):1676-1680.

25. Rosenberg, SA, Yang JC, Sherry RM, et al. Durable complete responses in heavily pretreated patients with metastatic melanoma using T cell transfer immunotherapy. Clin Cancer Res. 2011;17(13):4550-4557.

26. Gotherstrom C. Immuno- modulation by multipotent mesenchymal stromalcells. Transplantation. 2007;84(1 Suppl):S35-S37.

27. Loebinger MR, Janes SM. Stem cells as vectors for antitumour therapy. Thorax. 2010;65(4):362-369.

28. Menon LG, Picinich S, Koneru R, et al. Differential gene expression associated with migration of mesenchymal stem cells to conditioned medium from tumor cells or bone marrow cells. Stem Cells. 2007;25:520 528 .

29. Ringden O, Uzunel M, Rasmusson I, et al. Mesenchymal stemcells for treatment of therapy-resistant graft- versus-host disease. Transplantation. 2006;81(10):1390-1397.

30. Studeny M, Marini FC, Champlin RE, et al. Bone marrow-derived mesenchymal stem cells as vehicles for interferon-beta delivery into tumors. Cancer Res. 2002;62(13):3603-3608.

31. Sasportas LS, Kasmieh R, Wakimoto H, et al. Assessment of therapeutic efficacy and fate of engineered human mesenchymal stem cells for cancer therapy. Proc Natl Acad Sci USA. 2009;106(12) 4822-4827.

32. Eliopoulos N, Francois M, Boivin MN, et al. Neo-organoid of marrow mesenchymal stromal cells secreting interleukin-12 for breast cancer therapy. Cancer Res. 2008;68(12):4810-4818.

33. Xin H, Sun R, Kanehira M, et al. Intratracheal delivery of CX3CL1expressing mesenchymal stem cells to multiple lung tumors. Mol Med. 2009;15(9-10):321-327.

34. Uchibori R, Okada T, Ito T, et al. Retroviral vector-producing mesenchymal stem cells for targeted suicide cancer gene therapy. J Gene Med. 2009;11(5):373-381

35. Gu C, Li S, Tokuyama T, et al. Therapeutic effect of genetically engineered mesenchymal stem cells in rat experimental leptomeningeal glioma model. Cancer Lett. 2010;291(2):256-262. 